\title{
Neutron-Based Land Mine Detection System Development \\ Title:
}

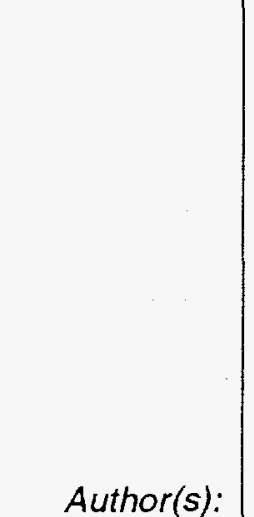

Author(s): Submitted to:
Harold A. Davis, P-24

Thomas E. McDonald Jr., P-23

Richard A. Nebel, T-15

Mark M. Pickrell, NIS-5
巽

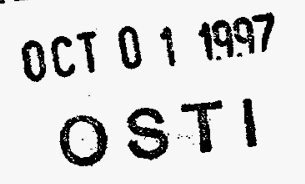

.

DOE Office of Scientific and Technical Information (OSTI)
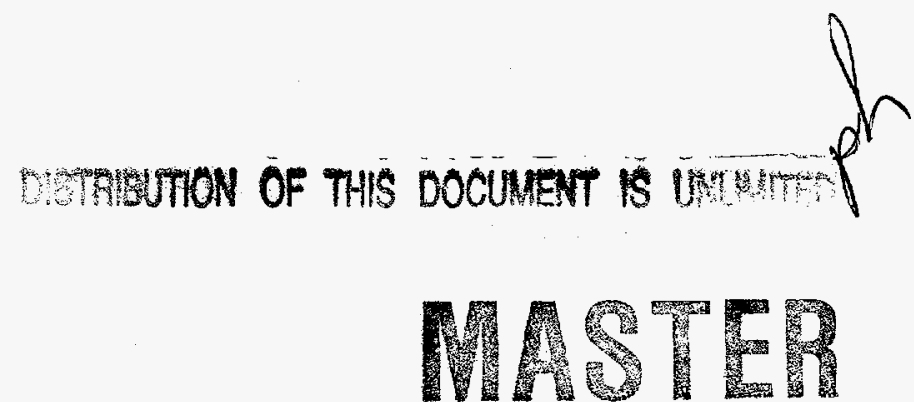

\section{Los Alamos}

Los Alamos National Laboratory, an affirmative action/equal opportunity employer, is operated by the University of California for the U.S. Department of Energy under contract W-7405-ENG-36. By acceptance of this article, the publisher recognizes that the U.S. Government retains a nonexclusive, royaltyfree license to publish or reproduce the published form of this contribution, or to allow others to do so, for U.S. Govemment purposes. Los Alamos National Laboratory requests that the publisher identity this article as work performed under the auspices of the U.S. Department of Energy. Los Alamos National Labotatory strongly supports academic freedom and a researcher's right to publish; as an institution, however, the Laboratory does not endorse the viewpoint of a publication or guarantee its technical correctness. 


\section{DISCLAIMIER}

Portions of this document may be illegible in electronic image products. Images are produced from the best available original document. 


\section{DISCLAIMER}

This report was prepared as an account of work sponsored by an agency of the United States Government. Neither the United States Government nor any agency thereof, nor any of their employees, make any warranty, express or implied, or assumes any legal liability or responsibility for the accuracy, completeness, or usefulness of any information, apparatus, product, or process disclosed, or represents that its use would not infringe privately. owned rights. Reference herein to any specific commercial product, process, or service by trade name, trademark, manufacturer, or otherwise does not necessarily constitute or imply its endorsement, recommendation, or favoring by the United States Government or any agency thereof. The views and opinions of authors expressed herein do not necessarily state or reflect those of the United States Government or any agency thereof. 


\title{
Neutron-Based Land Mine Detection System Development
}

\author{
Harold A. Davis, Thomas E. McDonald Jr.*, \\ Richard A. Nebel and Mark M. Pickrell
}

\begin{abstract}
This is the final report of a one-year, Laboratory-Directed Research and Development (LDRD) project at the Los Alamos National Laboratory (LANL). The goal of this project was to examine the feasibility of developing a land mine detection system that can detect nonmetallic (plastic) mines using the detection and analysis of prompt gamma-rays induced by neutron interrogation. We refer to this concept as prompt gamma neutron activation analysis (PGNAA). We approached this study by first carrying out a review of other nonmetallic land mine detection methods for comparison with the PGNAA concept. We reviewed issues associated with detecting and recording the return gamma signal resulting from neutrons interacting with high explosive in mines and we examined two neutron source technologies that have been under development at Los Alamos for the past several years for possible application to a PGNAA system. A major advantage of the PGNAA approach is it's ability to discriminate between explosive and nonexplosive objects. Disadvantages include slow detection speed and need for close proximity. We identified approaches to solving these problems through development of improved neutron sources and detection sensors.
\end{abstract}

\section{Background and Research Objectives}

The detection of buried land mines is a pressing issue worldwide. Both in the tactical and the humanitarian context land mines are a present threat. During the Gulf War, armored vehicles were destroyed by buried land mines in supposedly cleared breach lanes. A picture of a Marine Corps LVTP destroyed by mines is shown in Figure 1. Similarly, land mines have been deployed extensively in the third world, and represent a great danger to the indigenous civilian population. The immediacy of the problem is due to an estimated 80 to 100 million mines deployed worldwide, with several million more being deployed each year. These mines are causing an estimated several thousand casualties annually. The importance of this issue is evident from a DoD Broad Agency Announcement in response to the US Congressional directive to pursue the development of advanced countermine and demining technologies [1].

*Principal Investigator, E-mail: mcdonald@lanl.gov 
Mine detection research has been in progress for several years, and many different technologies have been explored. Each of the technologies has strengths and weaknesses. In our study, we performed a thorough evaluation of all the technologies to determine which ones had promise. We found two general classes of mine detection techniques. One type is very fast and can cover a broad area, but is not very accurate. Examples are ground penetrating radar and dual-band infrared. These methods can detect buried objects quickly and occasionally at significant distance. However, neither can reliably distinguish buried explosives from other buried objects. The number of false-positives from these methods can be effectively overwhelming. By contrast, the method of prompt gamma neutron activation analysis (PGNAA) is very specific to and reliable for the detection of buried explosives. Its limitation is that it is slow and requires the instrumentation to be proximal with the explosive. The detector and neutron source must often touch the ground to detect a land mine, and the time for a measurement can be as long as 15 minutes. This time and distance is prohibitive.

The prompt gamma neutron activation analysis method works by interrogating a sample with neutrons. The neutrons cause the atoms to emit characteristic gamma rays, which are then measured by a gamma detector. The gamma ray energy is specific to each element. By measuring the gamma rays, the elemental composition is determined. Buried explosives have a unique elemental composition that can be identified.

The other criterion we applied in our technology survey was to determine which methods had the potential for a significant increase in capability. The PGNAA method satisfied this requirement. The method is already accurate and reliable, and there is a direct path for a substantial reduction in measurement time (a nominal factor of 500) and an increase in distance, by a factor of at least 10 . The basis for this improvement was to exploit unique Los Alamos technology. The fundamental reason for the present limitation of the PGNAA approach is the gamma-ray data rate. In order to unambiguously identify an explosive, a sufficient number of gamma rays must be collected to uniquely determine the elemental composition of the object. Typically, nitrogen is the element of interest because it is present in all explosives in high concentration. Sufficient gamma rays must be detected to overcome counting statistics and precisely identify the elemental composition. The counting statistics problem is exacerbated by gamma rays from other elements and the Compton continuum. In order to speed the identification of the explosive, the rate at which the characteristic gammas are detected must be increased. There are two reasons that present systems have a limited gamma ray detection rate: the intensity of the interrogating neutron source and the speed of the gamma detectors. 
A more intense neutron source induces gamma rays at a faster rate. Existing PGNAA systems typically use californium sources with strengths of $10^{6}$ neutrons per second. Larger isotopic sources are not practical because of safety considerations. A more effective approach would be a high intensity neutron generator that could produce a much greater number of neutrons but could be turned off for safety. However, existing neutron generators of the size needed are too large, costly, heavy, and expensive. Moreover, their operating lifetime is limited to a few hundred hours, which is also not practical.

The second necessary component is a fast gamma detection system. If an intense neutron source is used for interrogation, a high rate of gammas is produced. These can saturate existing gamma ray detector systems. In order to capture all of the information in the gamma signature, faster detector systems are necessary.

The combination of a high intensity neutron source and faster gamma ray detectors could advance the PGNAA method from a very accurate system, to a system that is accurate, fast, and can operate at a modest distance from the explosive. Because we could identify a means to achieve this performance, we selected the PGNAA method as ideal for land mine detection. The objective of our research effort was to design a neutron source that would operate at $10^{11} \mathrm{n} / \mathrm{s}$ in a form factor for existing $10^{8} \mathrm{n} / \mathrm{s}$ systems. This source would be a factor of 100,000 more intense than typical $10^{6}$ isotopic systems. It would also have a long operating lifetime. We also investigated the possibility of fast gamma-ray detectors and possible methods for implementing this approach.

The specific objectives and deliverables for this research project were:

- Review current detection technologies for nonmetallic land mine detection.

- Develop a base-case design for the two high intensity neutron sources that were considered in the study: the Inertial Electrostatic Confinement (IEC) source and the Intense Ion-Beam (IIB) source.

- Develop general approaches for the development of fast gamma detectors.

- Evaluate the requirements for PGNAA to be an effective land mine detection system.

- Complete quantitative analysis of the PGNAA with Monte Carlo modeling to determine the exact performance requirements for this system.

All of these objectives were achieved in this project. 


\section{Importance to LANL's Science and Technology Base and National R\&D Needs}

This project leverages existing laboratory capabilities into a new application. The

Los Alamos core competencies that are addressed are: complex experimentation and measurement, analysis and assessment, and nuclear science, plasmas, and beams. This project directly supports the laboratory mission in conventional defense.

There were two major technical components to this project: 1) an assessment of the requirements and underlying physics for nonmetallic land mine detection with PGNAA and 2) determining how to solve the limitations of existing PGNAA systems using the unique capabilities of the laboratory. We have identified existing laboratory competencies that could be applied directly to solving this problem. The first competency is in the area of nuclear safeguards and security. The Los Alamos Safeguards program is in the forefront of developing state-of-the-art nuclear detection systems. One of the research areas in Safeguards is the development of new gamma-ray detectors. In particular, there is extensive research and modeling capability for new detector development. One case is the development of cadmium-zinc-tellurium (CdZnTe) detectors, which offer the possibility of medium-resolution, fast room-temperature detectors. At present, these are too small to be effective for the land mine problem, but the crystal sizes are continually increasing.

The second competency is in the area of plasma physics and plasma-based fusion. Los Alamos researchers have developed two concepts for the generation of high fluxes of neutrons. The two concepts are the triple-grid IEC plasma device and the IIB neutron source. Both machines have the potential of producing the required $10^{11} \mathrm{n} / \mathrm{s}$ in a compact form-factor. Los Alamos developed these innovative concepts and has the sophisticated plasma kinetic codes to simulate and evaluate the operation of the devices.

The evaluation that we completed has shown how Los Alamos can continue to contribute to the conventional defense, using largely existing technologies. We have pursued follow-on funding and two of our proposals have been funded: one by DOE/EM and one by DOE/ER. In addition, a joint STTR proposal with the University of Florida is in progress to develop a multisensor mine-detection system.

\section{Scientific Approach and Accomplishments}

The basic concept of the PGNAA is to interrogate a suspected area with neutrons and measure the gamma rays resulting from the neutron reactions with elemental constituents of the area. The explosive in mines has an elevated presence of nitrogen while the surrounding earth generally contains little or no nitrogen $[2,3]$. Some of the neutrons react with the nitrogen in the explosive and gamma rays having specific and unique energy 
are emitted as a result of the reaction. One primary reaction to be considered is neutrons with nitrogen-14, which produces nitrogen-15 and a 10.8-MeV gamma [3,4]. Detection of this $10.8-\mathrm{MeV}$ gamma is an indicator of the presence of explosive.

There have been several previous attempts to use the neutron-based (NB) technology for mine detection; however, most other attempts have relied upon spontaneous fission sources $[5,6,7]$. The difficulty with spontaneous fission sources (such as a californium source) is that only a relatively low-intensity neutron flux (in the range of $10^{6}$ to $10^{7} \mathrm{n} / \mathrm{s}$ ), together with heavy shielding, can be used because of personnel safety considerations [8]. A system using such a low intensity source is slow (several minutes) and requires close proximity (within a few centimeters) to the explosive being detected. Most studies indicate that a source strength in the range of $10^{9}$ to $10^{11}$ would be necessary to achieve a relatively high signal-to-noise ratio having a reliable detection capability that requires interrogation time of a few seconds $[9,10]$.

In this project we considered two alternative neutron sources that have the potential of neutron fluxes in the neighborhood of $10^{11} \mathrm{n} / \mathrm{s}$ : the Intense Ion-Beam (IIB) source [11] and the Inertial Electrostatic Confinement (IEC) source [12]. The advantage of these sources is that they can be turned off when not in use and can be operated over a wide range from steady state to pulsed. We also reviewed alternative detection techniques and carried out preliminary measurements using mock high explosive material. We pursued four areas of investigation in the study. The first area is the review of nonmetallic minedetection approaches, the second is a study of the detection issues to achieve an effective PGNAA system, the third and forth are the design of conceptual base-line designs of the two controlled neutron sources, the IIB and the IEC.

\section{A. Review of Nonmetallic Mine-Detection Approaches}

In this summary, we draw heavily upon a study report prepared by the Jet Propulsion Laboratory (JPL) for the US Army [13] and an earlier report summarizing the MERADCOM mine detection program for the period 1960-1980 [14]. The detection of explosive mines and unexploded ordnance waste is a problem that has challenged military technology developers since the advent of mine warfare. A large number of technologies and approaches have been evaluated in studies and experimentally for application to the mine detection problem. A major problem in developing countermine technologies is the wide spectrum of mine types that are encountered in size, shape, casing material, and explosive type. Technology has advanced to the point that detection of metallic mines, that is mines that contain significant quantities of metal such as a metal case, can be 
accomplished relatively reliably. However, metal nonexplosive objects such as cans and other debris will also be detected and result in signal noise, clutter, and false positives.

With the advent of plastic mines, which have little or no metallic components, metal mine detecting technologies must be supplemented with technologies that are effective in detecting nonmetallic mines. At present, no nonmetallic mine detecting technology fully meets the requirements of reliably detecting mines and all have their advantages, disadvantages, and trade-offs.

A selected list of the classes of technologies that have been used or considered for land mine-countermine applications is given in Table I.

\section{Table I. Listing of Countermine Technologies \\ Magnetometer/Gradiometer \\ Electromagnetic Induction \\ Ground-Penetrating Radar (GPR) \\ Visible Imaging \\ Infrared (IR) Radiometry and Spectrometry \\ Millimeter Wave (MMW) Radiometry \\ LIDAR (2- and 3-dimensional) \\ Nuclear Technology \\ Cone Penetrometers \\ Acoustic Sensors \\ Biological Sensors}

Of those listed above, the technologies that appear to have received the most attention and seem most promising for plastic mine detection are:

- Ground-Penetrating Radar (GPR)

- Infrared Detection ( 7 to 15 micorns)

- Metal Detection (set to detect firing pin)

- Electromagnetic Detection

- Nuclear Technologies: neutron-based

A brief summary of these technologies listing advantages and disadvantages follow.

Ground-Penetrating Radar. The GPR system basically uses the principle of radar to detect buried objects. The system illuminates the terrain with electromagnetic energy, detects signals reflected from objects in the ground, and records the signals as an image. In practice, a number of factors affect the performance of a GPR system. These include the type and moisture content of the soil in which the object to be detected is buried, the frequency of the electromagnetic wave used by the GPR system, and the electrical characteristics of the objects to be detected. Also, the system does not discriminate between mines and inert objects such as rocks, blocks of wood, and trash. Nonetheless, several mine detection systems based on GPR have been developed, fielded and used successfully. GPR systems can be made small enough to be person portable, although a 
full system tends to be heavy for a person. Larger systems can be used on airborne platforms. Advantages and disadvantages are given below.

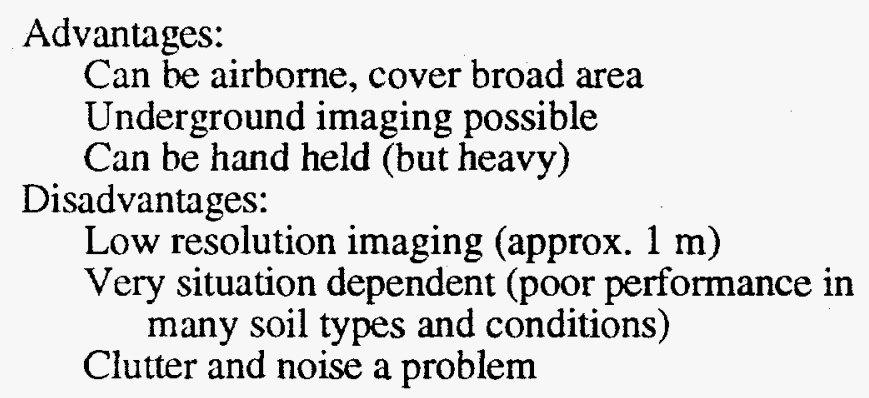

Infrared Detection ( 7 to 15 microns). Infrared (IR) radiometry is the technique of identifying objects by measuring their thermal energy signatures in the IR spectrum. Ordnance, whether exposed on the surface of the ground or buried, possesses a heat capacity that results in a different temperature from the surrounding soil. This temperature difference in turn results in a photon emittance form the ordnance (or mines) that can be differentiated from the emittance of the soil. Thus, the emissions from mines can be differentiated from the background emissions of the soil by an imaging IR detector array, which allows the approach to be used to detect the presence and location of mines, or mine-like objects. The technique is, however, sensitive to nonexplosive objects resulting in noise and clutter in the data. Successful detection using this approach depends on adequate mine-to-soil contrast, which is affected by a number of parameters and conditions. Weather conditions, time of day, background environment, and size and composition of the mines are major factors in achieving a good detection signal. The mineto-background contrast can be improved by using multiple IR bands, which is a technique referred to as IR spectrometry. Although the approach has been demonstrated as a mine detector, at least one report has expressed the opinion that, although the IR sensors perform well, the technique is not as well suited to detecting buried mines as a sensor with groundpenetrating capability such as a GRP.

\section{Advantages}

Can be airborne; cover broad areas

Person portable capability

Provides images of mine layout

Disadvantages

Very situation dependent (most successful during thermal transitions such as at sunrise or sunset)

Limited to "good weather" (no fog, rain, etc.)

Clutter and noise a problem 
Electromagnet Detection. This is a relatively new concept being investigated by Los Alamos for application to the mine detection problem. The approach has not had extensive development for the countermine application; however, it appears promising for the sensing of nonmetallic mines. The concept is relatively straightforward. An electromagnetic wave is established by an antenna near the surface of the soil to be examined. The amplitude of the field coupled between the antenna and soil depends upon, among other things, the electrical properties of the soil. When an object, such as a mine, is buried in the soil, these electrical properties are modified from that of the soil itself and the amplitude of the coupled electromagnetic field changes. Thus, it is possible to sense the difference between homogenous soil and soil in which an object has been buried. A further refinement is to employ an array of antennas, which allows location and size of the buried object to be determined.

\author{
Advantages \\ Higher sensitivity than GPR \\ Person portable capability \\ Potential for imaging \\ Disadvantages \\ Clutter and noise a problem \\ Emerging technology (much development \\ required)
}

Sensitive Metal Detection. This is not a technique that can detect a fully nonmetallic mine per se. The approach relies on the fact that most mines contain some small amount of metal, for example, a firing pin. A good metal sensing mine detector can be set for high sensitivity and, with care and patience by the operator, can be used to detect the few ounces of metal that may be in a mine. The approach is advocated by some in the countermine community. Several potential difficulties can be identified, such as, detection requires a skilled technique by the operator and is time consuming, the approach is especially susceptible to debris and clutter in the soil, and the inability to detect all plastic mines. Nonetheless, this is a well developed and proven technology and under the appropriate circumstances can probably be used to good advantage.
Advantages
Mature technology in the field; troops familiar with and know how to use
Person portable capability
Disadvantages
Cannot detect completely nonmetallic mines
Clutter and noise a major problem
Slow and careful operation required 
Nuclear Technology. The nuclear technology concept is an approach whereby a region to be examined is interrogated with nuclear particles (such as neutrons, electrons, and protons) or with photons (gamma rays). These particles or photons interact with the elements to be identified on a nuclear level and particles or photons are scattered or emitted that are unique to the element being identified. Many possibilities exist and have been studied for interrogating particles or photons and for signature particles or photons. One of the more promising combinations that has received much attention from researchers is the use of neutron activation with ${ }^{14} \mathrm{~N}$ and detection of the unique $10.8-\mathrm{MeV}$ gamma ray resulting from the reaction. This reaction is attractive because almost all explosives contain a relatively high concentration of nitrogen and there is typically little or no nitrogen in the surrounding soil. Thus, a neutron-based detection system gives a signature of the explosive itself and is not plagued by other objects in the soil such as rocks or debris.

A disadvantage of the approach using present technology is that the neutron source is typically a spontaneous emission source such as californium, which must, because of personnel safety, have a relatively low intensity (typically around $10^{6} \mathrm{n} / \mathrm{s}$ ) and be well shielded, which significantly increases weight. Thus, in order to have sufficient neutron interactions, the source must be close to the object being examined and several seconds or minutes are needed to complete a reading. As a result, the technique has been developed for use in airports where close proximity to the objects being examined can be achieved and time is not as critical. Also the technique has, more recently, been developed by Idaho National Engineering Laboratory and commercialized by EG\&G Ortec for examination of unexploded ordnance, where an item being examined can be placed next to the source and detector, and sufficient time is available to carry out the reading.

Because of the weight penalty and low signal with present technology, the technique has not been successfully applied to the land mine detection problem. However, over the years researchers continue to return to the approach because of its unique explosive discriminator capabilities. In this project, we are examining high-flux, controlled neutron sources and improved detector technology to achieve an improvement in return signal strength by an estimated factor of $10^{5}$, which will allow the source and detector to be placed well away (feet or meters) from the examined area. As mentioned elsewhere in this report, we refer to this improved system as the PGNAA. This allows readings to be made within seconds and the signal may be sufficiently strong to allow imaging of areas. However, the system continues to be relatively large and heavy and will require a vehicle for transportation.

The advantages and disadvantages of a system with current technology and of a PGNAA system using the Los Alamos improvements are summarized below. 


\section{Advantages \\ Nuclear Technology: Current Technology System}

Strong discriminator for explosives

Disadvantages

Large and heavy; requires vehicle for transportation.

Slow; readings require seconds to minutes to complete.

Relatively expensive (hundreds of thousands to millions of dollars)

Requires close proximity to object being examined (centimeters)

Nuclear Technology: Improved PGNAA System Projected

Advantages

Technique a strong discriminator for explosives

Significant stand-off possible (feet or meters)

Readings completed within seconds

Potential for imaging

Disadvantages

Large and heavy; requires vehicle for transportation

Relatively expensive (hundreds of thousands to millions of dollars)

Although the PGNAA system shows significant promise, we do not project that it will replace existing systems. We propose that the PGNAA be used with other technologies in a suite of complementary detectors that will cover a broad area around the transporting vehicle. The PGNAA would be used as a discrimination tool between explosive objects and nonexplosive clutter and debris.

\section{B. Return Signal Detection}

An important component of this study was to quantitatively assess the required gamma-ray detection rate and the interrogating neutron source rate necessary to make the PGNAA method effective and to determine technologies that could implement it . The initial proposal called for extensive MCNP modeling of the coupled neutron/gamma-ray problem to determine what intensity of neutron source was necessary for effective de-mining technology. However, we compared initial MCNP runs against experimental measurements to benchmark our models. The MCNP calculations did not agree with experiment at all. We investigated this discrepancy and soon found that the libraries in MCNP are incomplete for the neutron-induced gamma-ray process. It would not be possible to use MCNP for this purpose.

We chose as an alternative to work from existing neutron-based systems. The most successful and effective, in our judgment, is the Portable Isotopic Neutron Spectroscopy (PINS) system developed by the Idaho National Engineering Laboratory (INEL) and produced by EG\&G ORTEC [15]. The PINS system uses a modest californium source 
$\left(10^{6} \mathrm{n} / \mathrm{s}\right)$ and a high purity germanium (HPGe) detector. PINS has successfully measured 155 millimeter munitions and determined whether they were explosive, chemical agent or smoke. Moreover, PINS can determine the type of chemical agent or explosive. Typical PINS measurements take 15 minutes and the detector touches the sample.

Although we could not use the MCNP calculations to model, in entirety, the land mine detection problem, we were able to use MCNP to scale from the PINS results. The number of induced gamma rays per source neutron from a 600 gram sphere of "Composition B" type explosive was determined and several different spectrum tailoring configurations were tried using different thicknesses of polyethylene. Two different depths were tested. The source was located 2 meters above the ground and the ground was modeled as diatomaceous earth. In both cases the nominal response is $2 \times 10^{-6}$ gammas per source neutron. Using similar conditions, we also modeled the PINS system. The response was $3.2 \times 10^{-5}$. The PINS model assumed a californium spectrum and the MCNP calculations assumed a moderated deuterium-tritium spectrum. The PINS response is a factor of 16 larger. From these results it is possible to calculate the required strength of the neutron source. The various terms are given in Table II.

\section{Table II. Summary of Factors Used in Determining Required Neutron Source Flux}

\begin{tabular}{ll}
\multicolumn{1}{c}{ Description of Term } & \multicolumn{1}{c}{ Value } \\
Change in neutron interrogation from & 16 \\
distance. & \\
Change in gamma ray intensity from distance & 16 \\
Reduction in measurement time & 1000 \\
Net increase required & 250,000
\end{tabular}

The reduction of measurement time is based on a nominal 1,000 second (approximately 15 minute) measurement time for PINS. The factor of 16 for the neutron interrogation flux was determined from the MCNP calculations. The second factor of 16 was due to inverse square scaling of the gamma-ray intensity with distance. The net increase in neutron intensity necessary for a fast measurement at a modest distance is roughly $10^{5}$. Existing PGNAA neutron sources are $10^{6} \mathrm{n} / \mathrm{s}$; therefore, the source strength that we calculate should be $10^{11} \mathrm{n} / \mathrm{s}$. This value was used in developing the base-line designs of the two neutron sources. 
The second component of the physics research was determining the means for increasing the gamma-ray detection rate. We determined that several methods could be tried, that in combination could increase the detection rate by the necessary amount. In order to reduce the assay time by a factor of 1,000 , the gamma ray detection rate must be increased by the same factor. Some of the strategies that we have considered are: using multiple detectors, using detectors with a "Compton" shield, using correlated gammas from the cascade process in the isotope decay, and using CdZnTe detectors. Several approaches were suggested in the follow-on proposal [16].

\section{Intense Ion Beam Neutron Source}

\section{Background}

Over the past two decades researchers in the US, Germany, Russia, and Japan have been investigating the application of single-shot, intense, pulsed-ion-beam technology $(\mathrm{E}=$ $1-30 \mathrm{MeV}, \mathrm{I}=0.1$ to $1 \mathrm{MA}, \mathrm{t}=10-50 \mathrm{~ns}$ ) to inertial confinement fusion programs. Over the past decade, using more modest beam parameters ( $E=0.1-1.0 \mathrm{MeV}, \mathrm{I}=0.005-0.05$ $\mathrm{MA}, \mathrm{t}=100-1000 \mathrm{~ns}$ ), research into the processing of materials using this technology has emerged. Other applications such as high-flux neutron sources for nonmetalic mine detection appear attractive, but require repetitive rather than single-shot ion beam sources. In this project we have investigated the feasibility of developing such a source for the detection of nonmetalic mines.

\section{Operating Principle}

Intense ion beams are produced in magnetically insulated vacuum diodes, which require a source of ions, an accelerating voltage, and a magnetic field transverse to the acceleration gap to suppress electron flow and enhance the ion flow (Figure 2). Ion currents typically exceed the vacuum space-charge limit by 5-50 times owing to electrons confined in the acceleration region by the applied magnetic field. The beams are produced and transported in vacuum of at least $10^{-4}$ Torr. Traditionally ions are drawn from the surface of a polymer anode converted to a plasma by a combination of high-voltage flashover and electron impact. Polymer anodes are unacceptable for applications requiring repetitive operation because of limited lifetime, excessive heat loading, and high gas production. In our concept, ions are drawn from a pre-formed plasma of $\mathrm{D}^{+}$ions. The traditional single-shot beam accelerator (using Marx generators and high-voltage pulse lines), incompatible with repetitive operation, will be replaced by a thyratron-switched Blumlein line transmission charged to $60 \mathrm{kV}$ driving the input of a 5:1 step up transformer to achieve a $300 \mathrm{kV}$ accelerating pulse for one microsecond. Repetitive rates up to $30 \mathrm{~Hz}$ 
appear feasible. The $\mathrm{D}^{+}$ions are incident on a tritiated metalic target to produce $\mathrm{D}-\mathrm{T}$ fusion neutrons with 14-MeV energy.

The key element of this technology is the magnetically insulated extraction diode, which is shown in converging geometry in Figure 2. The anode consists of a flat pulsed induction coil in an aluminum housing. The high-voltage plasma formation coil is formed from a set of parallel multi-turn spiral windings coaxial with the system axis. The diode, shown in focused geometry in Figure 2, will be in unfocussed cylindrical geometry for the mine detection application. The plasma anode is formed by first radially ducting a puff of gas with a fast-acting valve (risetime $\sim 100 \mathrm{~ms}$ ) located on axis over the coil surface. When the gas puff is properly distributed, a fast-rising current pulse $\left(10-20 \mathrm{kA}, \mathrm{t}_{\text {rise }}=1-2 \mathrm{~ms}\right)$ delivered to the induction coil breaks the gas down and induces azimuthal current in the plasma at the coil surface. The $J_{\theta} \times B_{r}$ force on the plasma accelerates the plasma to the radial opening in the aluminum anode housing where it is stagnated against the applied radial magnetic field. Ions are accelerated from this location. The cathode consists of the tips of two thin concentric metal cylindrical sections. The gap between the cathode tips and the plasma anode is a few $\mathrm{cm}$. Before application of the induction coil and accelerating voltages, a $200-\mu$ s risetime magnetic field of about $1.5 \mathrm{kG}$ is applied transverse to the anode-cathode gap by two magnetic field coils -- one located inside the inner cone and one located outside the outer cone. At peak field and when the plasma is in position at the anode housing aperture, a positive accelerating voltage supplied from a high-voltage modulator is applied to the anode. The applied magnetic field strength is adjusted to prevent electrons from crossing the anode-cathode gap, but the more massive ions, only very weakly deflected by the magnetic field, have approximately linear trajectories. System Parameters

Diode: The conceptual design of a $10^{11}$ neutron/shot source has been performed. At $1 \mathrm{~Hz}$ this is a $10^{11} \mathrm{n} / \mathrm{s}$ source, but it is expected that extension to up to $30 \mathrm{~Hz}$ will be straightforward giving $3 \times 10^{12} \mathrm{n} / \mathrm{s}$. At $300-\mathrm{keV}$ ion energy, $2 \times 10^{-5}$ neutrons are generated for each ion incident on a solid tritiated target. If we make the very conservative assumption that only half the current is incident on the target, then $1.6 \mathrm{kA}$ of beam current is required or a beam energy of about $500 \mathrm{~J} /$ pulse. Assuming that the current density is ten times the Child-Langmuir limit for vacuum flow (typical values achieved are in the range of 5-50 owing to the presence of electrons confined in the acceleration gap) and that the beam is annular with the inner radius 0.75 times the outer radius, $r_{0}$, than $r_{0}=3.2 \mathrm{~d}$ where $\mathrm{d}$ is the acceleration gap spacing. Using the theory of Dejarlais [17] and 10 times the vacuum space charge limit, the relation between the applied magnetic field and $d$ becomes: $B \times d=0.6$ 
where $B$ is in $W / \mathrm{m}^{2}$ and $d$ is in $\mathrm{cm}$. Thus we have two equations and three unknowns and are free to select one of the three. An attractive design has $r_{0}=9 \mathrm{~cm}, \mathrm{~d}=2.1 \mathrm{~cm}$ and $\mathrm{B}=670$ G. The magnetic field is low enough that it may be possible to supply it with an array of magnets placed on the outside of the inner and outer cathode cylinders. This would eliminate the need for a pulsed magnetic field coil.

Targets: Assuming that the beam ions are incident on a $400 \mathrm{~cm}^{2}$ target, twice the anode emission area, then the $0.3 \mathrm{~J} / \mathrm{cm}^{2}$ flux incident on an Er target, for example, would lead to an instantaneous temperature rise on the target surface (i.e., the top 2 microns) of less than the desorption temperature of $\operatorname{Er}[18]$. The use of target cooling with water should maintain the steady-state temperature of the target at acceptable levels. Another important issue is the lifetime of the targets. Typical targets have a lifetime of $5 \mathrm{~mA}-\mathrm{hr} / \mathrm{cm}^{2}$ [19]. This compares with $5.6 \times 10^{-7} \mathrm{~mA}-\mathrm{hr} / \mathrm{cm}^{2}$ for a single pulse with our source giving $9 \times 10^{6}$ pulses before anode replacement. This translates into 2500 hours before anode replacement for $10^{11} \mathrm{n} / \mathrm{s}$ at $1 \mathrm{~Hz}$ to 250 hours of operation for $10^{12} \mathrm{n} / \mathrm{s}$ at $10 \mathrm{~Hz}$. Operation during daylight hours seven days a week would require target replacement at intervals of 3 to 30 weeks.

Electrical Design: The beam electrical system requires modulator subsystems synchronized with each other and the ion acceleration pulse. A gas puff modulator and the induction coil modulator subsystem will be housed in a "hotdeck" chassis at common potential with the pulsed anode. For electrical isolation, fiber optic cables will carry fast control and diagnostic signals. The accelerating power system will utilize a lumped element transmission line charged to $60 \mathrm{kV}$ and switched with an English Electric Valve CX1736AX dual-gap hollow anode thyratron capable of handling up to 50\% voltage reversal due to load mismatch. The $30-\mathrm{kV}$ output voltage will be stepped up to $300 \mathrm{kV}$ with a 10:1 step-up transformer. The transmission line will have 10 networks with total capacitance equal to $250 \mathrm{nF}$ and total inductance of $1000 \mathrm{nH}$. Assuming $50 \%$ electrical efficiency, power requirements for 1 and $10 \mathrm{~Hz}$ systems vary between about 1 and $10 \mathrm{~kW}$ requiring a modest portable generator for field deployment.

Physical Parameters: Very preliminary estimates of system size and weight have been made. The accelerator will be housed in a cubical tank approximately $5 \mathrm{ft}$ on a side. The diode and target region will be under vacuum in a cylindrical chamber having a diameter of 1.5 to $2 \mathrm{ft}$ and a length of 2.5 to $3.0 \mathrm{ft}$. In addition a vacuum pump will also have to be installed. To minimize weight the electrical system will be insulated with high pressure $\mathrm{SF}_{6}$ gas. This will require a heavy-walled metal tank but eliminates the need for oil insulation, which would add significant weight to the system. The overall system weight cannot be readily estimated, but would probably be a few thousand pounds. 


\section{IEC Neutron Source}

\section{Background}

The IEC device is a plasma confinement scheme for fusion applications based on electrostatic fields. Unlike conventional magnetic confinement fusion, IEC devices produce fusion via non-Maxwellian beam-beam interactions; the kinetic energy of the beam ions being approximately the same as the potential on the grid. The IEC approach has potential for immediate application as a portable neutron source. Steady-state neutron yields as high as $2 \times 10^{10} \mathrm{n} / \mathrm{s}$ have already been demonstrated [20]. Applications for neutron sources in this range include safeguards and proliferation, assaying of nuclear and chemical waste, well logging, and detection of high explosives such as land mines (the purpose of this study). These applications require a compact, portable, and inexpensive neutron source. We believe that IEC has significant advantages in terms of both cost and safety. Our contribution was to complete a preliminary design for a prototype portable IEC source capable of delivering $1 \times 10^{11} \mathrm{n} / \mathrm{s}$ steady state.

\section{IEC Source Design Parameters}

Source parameters have been scaled from Hirsch's data [20]. Pessimistic and optimistic scalings have been assumed in order to bracket the expected performance of the device. Experimental parameters have been chosen so that the desired plasma parameters can be achieved even under the pessimistic assumptions. Figure 3 shows the projected current vs voltage curves required for a $1 \times 10^{11}$ source asuuming that the neutron yield scales like the current (top curve) or as the current squared (bottom curve). We have sized our power supplies at $75 \mathrm{KV}$ and $335 \mathrm{~mA}$, which should allow us to produce a $1 \times 10^{11}$ source even if the pessimistic assumptions prevail.

Figure 4 shows a conceptual layout for the three grids in the system. The outer two grids, in conjunction with electron guns on the boundary and a neutral fill gas, serve as a spherical ion source for the device. The inner grid then accelerates these ions and focuses them into a spot in the center. Table III is a summary of the device parameters.

\section{Table III. Summary of IEC Base-Line Design Parameters}

$\begin{array}{ll}\text { Chamber diameter } & 12 \text { inches } \\ \text { Number of grids } & 3 \\ \text { Number of e-guns } & 6 \\ \text { Current per e-gun } & 1 \mathrm{~A} \\ \text { Maximum grid voltage } & 75 \mathrm{KV} \\ \text { Maximum current rating } & 335 \mathrm{~mA} \\ \text { Anticipated fill pressure } & 0.1 \mathrm{mtorr} \\ \text { Anticipated base pressure } & 10^{-8} \text { torr } \\ \text { High voltage feedthroughs } & 2\end{array}$


The front view of the chamber layout is shown in Figure 5, which includes all of the ports and their purposes. The chamber is disassembled along its equator, which is how the grids would be assembled and accessed in the device. The top and bottom halves of the chamber are identical. They are rotated 60 degrees with respect to each other so the e-guns sit on the faces of a cube. Since the device has up to $25 \mathrm{~kW}$ of continuous input power, active cooling is required. The chamber shell is a double jacketed design with the coolant flowing between the chamber wall and the outer jacket. The high voltage feedthrough port is cooled along with the chamber.

We are fabricating this device under a follow-on project. It is anticipated that this device will operate in a hot cell at Los Alamos. Phase I operation will be with deuterium in a continuous feed pumped system. Optimal operational parameters will be determined during this phase. Phase II operation will be with tritium in a hermetically sealed gettered device. Full neutron production is anticipated during this phase.

\section{E. Summary of Results}

This project accomplished it's goals and objectives and included the following accomplishments:

- Reviewed nonmetallic mine-detection approaches.

- Reviewed literature on neutron-based detection.

- Initiated modeling of neutron-based configuration on MCNP.

- Initiated experiments using mock high-explosive and available neutron sources.

- Developed conceptual design of neutron sources to provide $10^{11} \mathrm{n} / \mathrm{s}$ pulsed or steady state for both the Inertial Electrostatic Confinement and Intense Ion Beam source concepts.

- Established contacts and interactions with program managers and researches in the mine-detection community.

- Two follow-on projects resulted from this LDRD project and a joint STTR proposal with the University of Florida is in progress.

The advantage of the neutron-based mine-detection approach is that the approach is a powerful discriminator. Other approaches, such as ground penetrating radar and infrared detection can detect buried objects; but these objects may or may not be mines. The neutron-based approach, on the other hand, detects explosive and differentiates between inert objects and explosive-containing mines. The problem is that the technique is slow and requires close proximity to the object being detected. Our approach of using a controlled, high-flux neutron source increases the speed of detection and allows a longer stand-off 
distance. In this new concept, however, weight and size are not significantly reduced, a vehicle is needed to transport the system, and costs are projected to be relatively high compared to more conventional mine detection techniques.

We propose that the neutron-based approach be used in conjunction with other approaches in a suite of complementary detection sensors. For example, infrared detection could be used to cover ground at a distance in front of the detecting vehicle, ground penetrating radar used to detect specific buried objects near the vehicle, and the PGNAA used to discriminate between inert objects and mines.

\section{Publications}

1. M. Pickrell, Neutron Based Land Mine System Development, Final Report, LANL LDRD Project Number: 96-508, 1996.

2. Los Alamos National Lab Proposal \# R-1835-97-0, "Development of a High Fluence Neutron Source and Fast Gamma Detectors for the Detection of Buried Land Mines", submitted to U. S. Army Communications \& Electronics Command Night Vision \& Electronic Sensors Directorate.

3. H. A. Davis, et al., Intense Ion Beam Treatment of Materials, Bulletin of the materials Research Society, Vol. 21-8, 1996 (LAUR-96-1450).

4. H. A. Davis, et al., Progress Toward a Microsecond Duration, Repetitive, Intense Ion Beam, Review of Scientific Instruments, 1996 (LAUR-96-1648)

\section{References}

1. Broad Agency Accouncement, Countermine for Demining in Operation other than War, Solicitation DAAB12-95-BAA1, Issued by Night Vision and Electronic Sensors Directorate AMSEL-RD-NV-MD (Briggs), Fort Belvoir, Virginia, Dec. 9, 1994.

2. Robert S. Carmichael, editor, Handbook of Physical Properties of Rocks, Vol I, CRC Press, Inc. Boca Raton, Florida, 1982.

3. J. G. Campbell, Landmine Detection by Scatter Radiation Radiography, Dissertation, University of Florida, Gov. Accession No. AD-A182227, July 1987.

4. T. J. Kennett, W. V. Prestwich, and J. S. Tsai, The ${ }^{14} \mathrm{~N}(\mathrm{n}, \gamma){ }^{15} \mathrm{~N}$ Reaction as Both an Intensity and Energy Standard, Nuclear Instruments and Methods in Physics Research, A249, 1986.

5. W. A. Coleman, R. O. Ginaven, and G. M. Reynolds, Nuclear Methods of Mine Detection, Vol I, Final Technical Report, SAI 74203 LJ, Science Applications, Inc. La Jolla, CA, 1974.

6. W. A. Coleman, R. O. Ginaven, and G. M. Reynolds, Nuclear Methods of Mine Detection, Vol III, Final Technical Report, SAI 74203 LJ, Science Applications, Inc. La Jolla, CA, 1974. 
6. W. A. Coleman, R. O. Ginaven, and G. M. Reynolds, Nuclear Methods of Mine Detection, Vol III, Final Technical Report, SAI 74203 LJ, Science Applications, Inc. La Jolla, CA, 1974.

7. R. B. Moler, Workshop Report Nuclear Techniques for Mine Detection Research, Sponsored by Belvoir Research and Development Center, Lake Luzerne, NY, July 1985.

8. S. I. Taimuty, An Experimental Study of Mine Detection Methods Based on Neutron Scattering, Final Technical Report, Stanford Research Institute Project Number PU828, for Engineer Research and Development Laboratories, Fort Belvoir, Virginia, October 1954.

9. J. J. Walker, Final Report of Project: Stand-off Mine Detector, Amparo Corp., US Army Missile Command, Contract DAAH01-89-C-0493, January 1991.

10. J. J. Walker, M. M. Hoffman, D. G. Rickel, Final Technical Report (Stand-Off Mine Detector), Defenxe Advanced Research Projects Agency, ID No. DAAH01-91-PR002, January 1991.

11. D. J. Rej, R. R. Bartsch, H. A. Davis, R. J. Faehl, J. B. Greenly, and W. J.

Waganaar, Microsecond Pulse Width, Intense, Light-ion Beam Accelerator, Rev. Sci. Instrum. 64 (10), October 1993.

12. D. C. Barnes, R. B. Nebel, Portable, Lightweight Neutron Source for Mine Detection, NambeTech, Inc. under contract DAAK70-93-C-0038, for U.S. Army Belvoir Research, Development, \& Engineering Center, January 1994.

13. J. C. Peterson, et al., Sensor Technology Assessment for Ordnance and Explosive Waste Detection and Location, Jet Propulsion Laboratory Report: JPL D-11367 Rev B, March 1995.

14. R. V. Nolan, et al., MERADCOM Mine Detection Program: 1960 - 1980, Report No. AD C021731, U.S. Army Mobility Equipment Research and Development Command, Fort Belvoir, Virginia, March 1980.

15. Portable Isotopic Neutron Spectroscopy Chemical Assay System, INEL and EG\&G ORTEC CRADA 93-ST-12, Company Brochure, Ph: (800) 251-9750, 1996.

16. Los Alamos National Lab Proposal \# R-1835-97-0, "Development of a High Fluence Neutron Source and Fast Gamma Detectors for the Detection of Buried Land Mines", submitted to U. S. Army Communications \& Electronics Command Night Vision \& Electronic Sensors Directorate, 1997.

17. Dajarlais, Phys. Fluids B 8, 1709 (1989).

18. J. Csikai, CRC Handbook of Fast Neutron Generators, CRC Press, Boca Raton Fl, V1 p. 77.

19. Ibid, V1 p. 76.

20. R. L. Hirsch, J. Appl. Physics $\underline{38}, 4522$ (1967). 


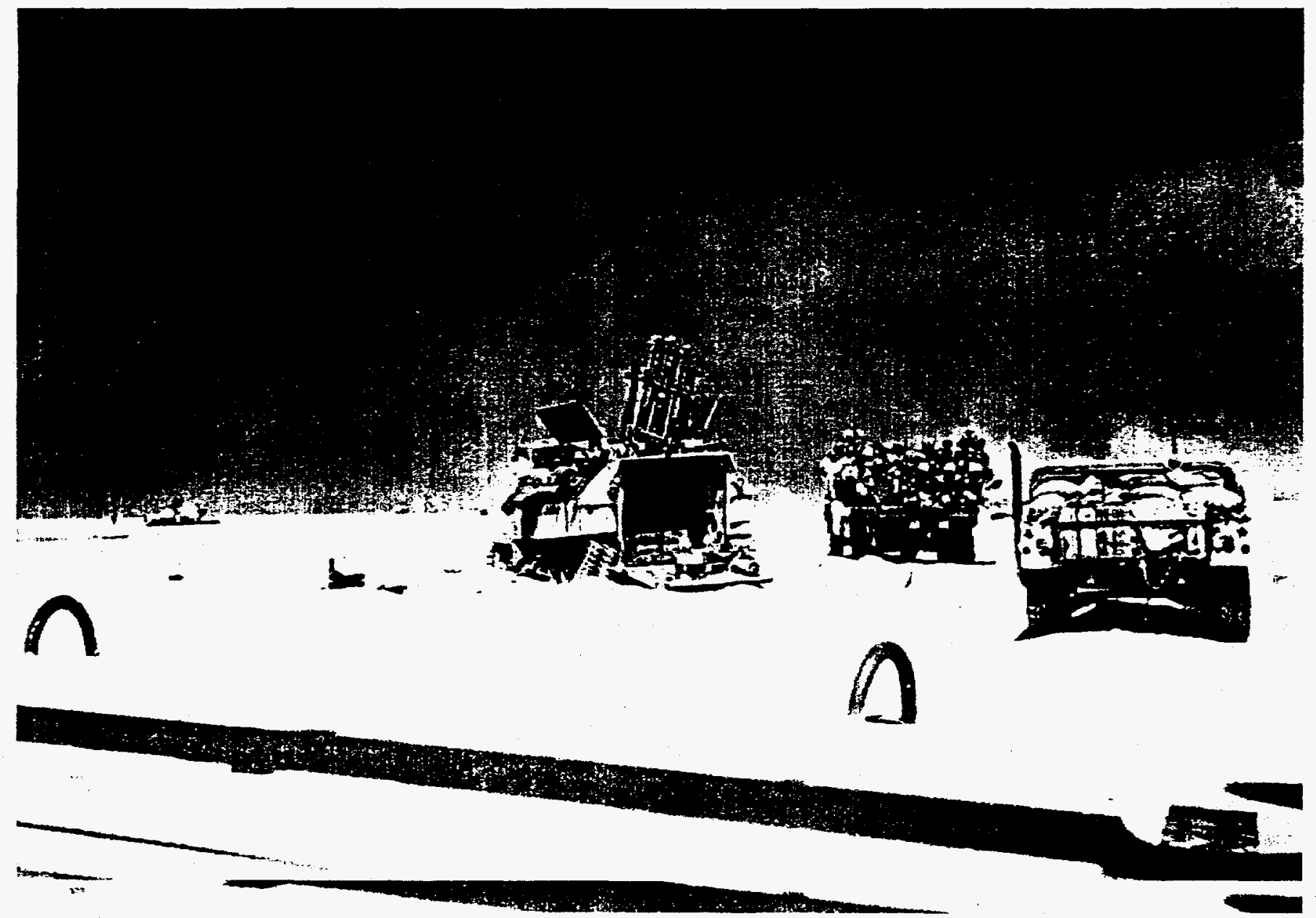

Figure 1. A picture taken by one of the authors (Pickrell) of the effects of a buried land mine during the Gulf War. The picture is of a Marine Corps LVTP that was destroyed in the initial ground assault into Kuwait. The location is breach lane "Green 6" on the right flank of the 2nd Marine division, near the Um Gudair oil field in southern Kuwait, during the first few minutes of the ground war. 


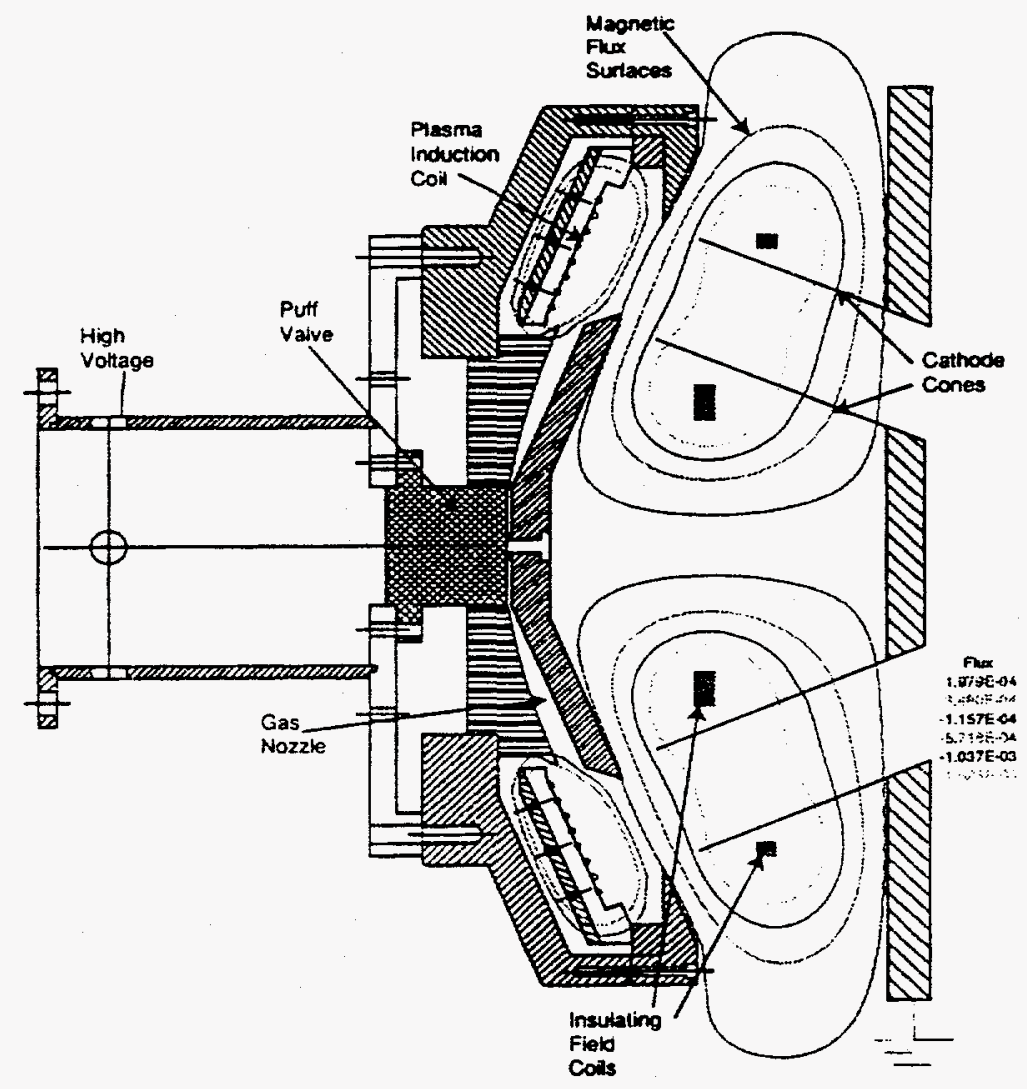

Figure 2. Layout of Intense Ion Beam neutron source diode. 


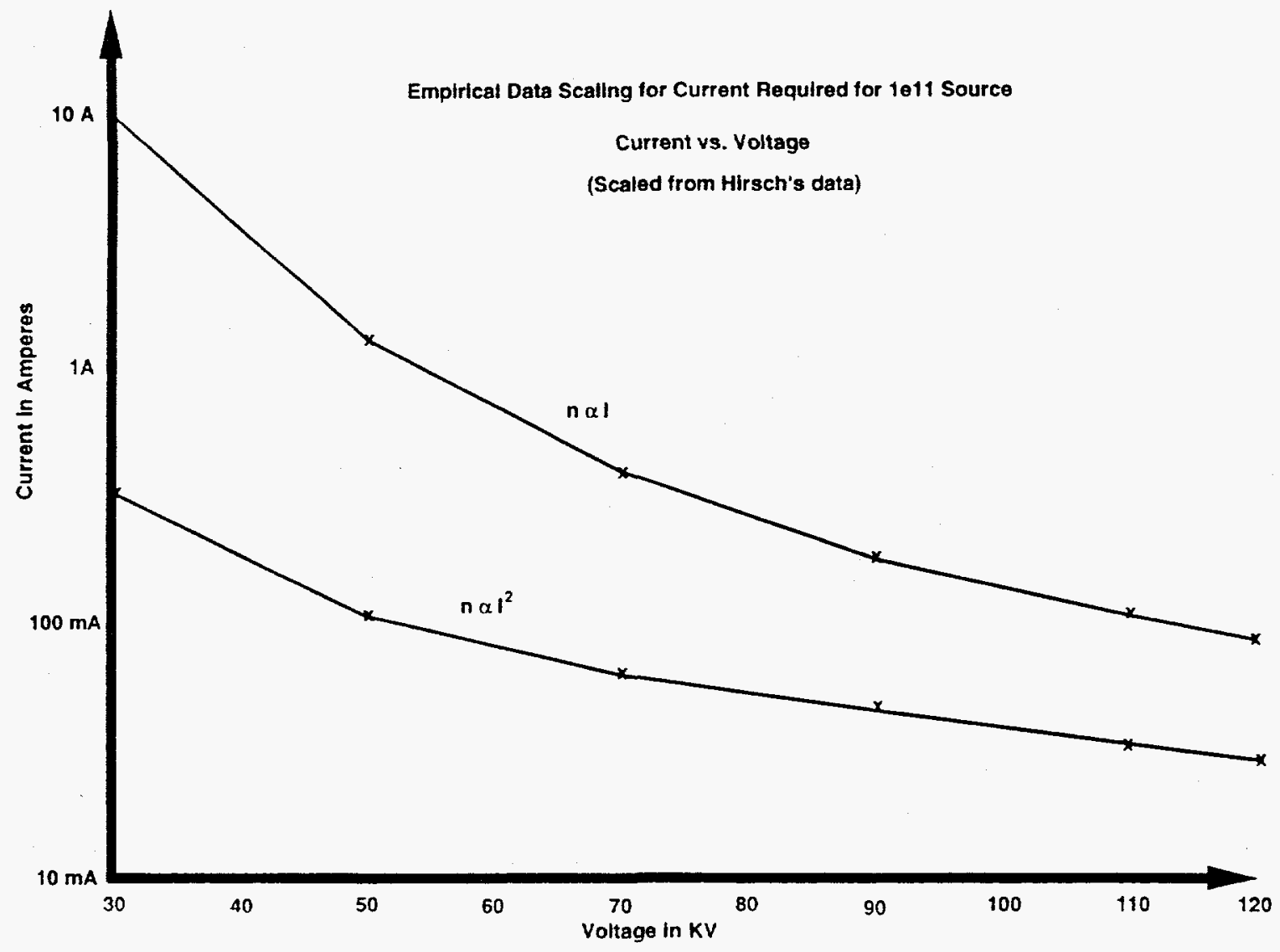

Figure 3. Empirical data scalling for current required for $10^{11}$ IEC neutron source. 


\section{IEC Neutron Source (1e11 n/second)}

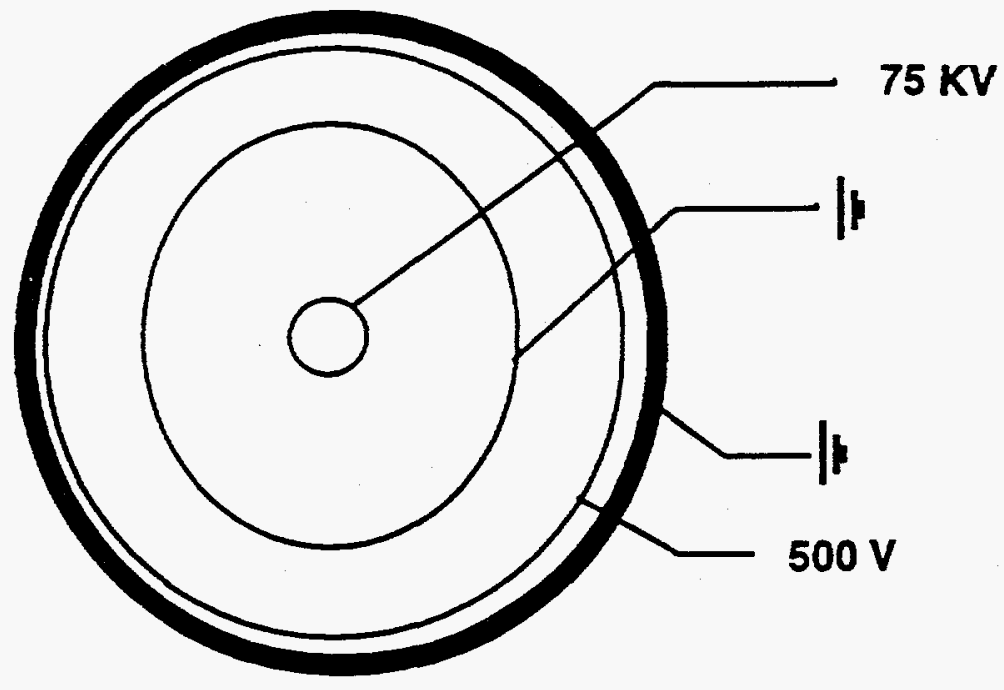

$$
\begin{aligned}
& \text { Parameters } \\
& d=12 \text { inches } \\
& p=0.1 \mathrm{mtorr} \\
& V=75 \mathrm{KV} \\
& I=335 \mathrm{~mA}
\end{aligned}
$$

Figure 4. Schematic diagram of base-line design IEC neutron source. 


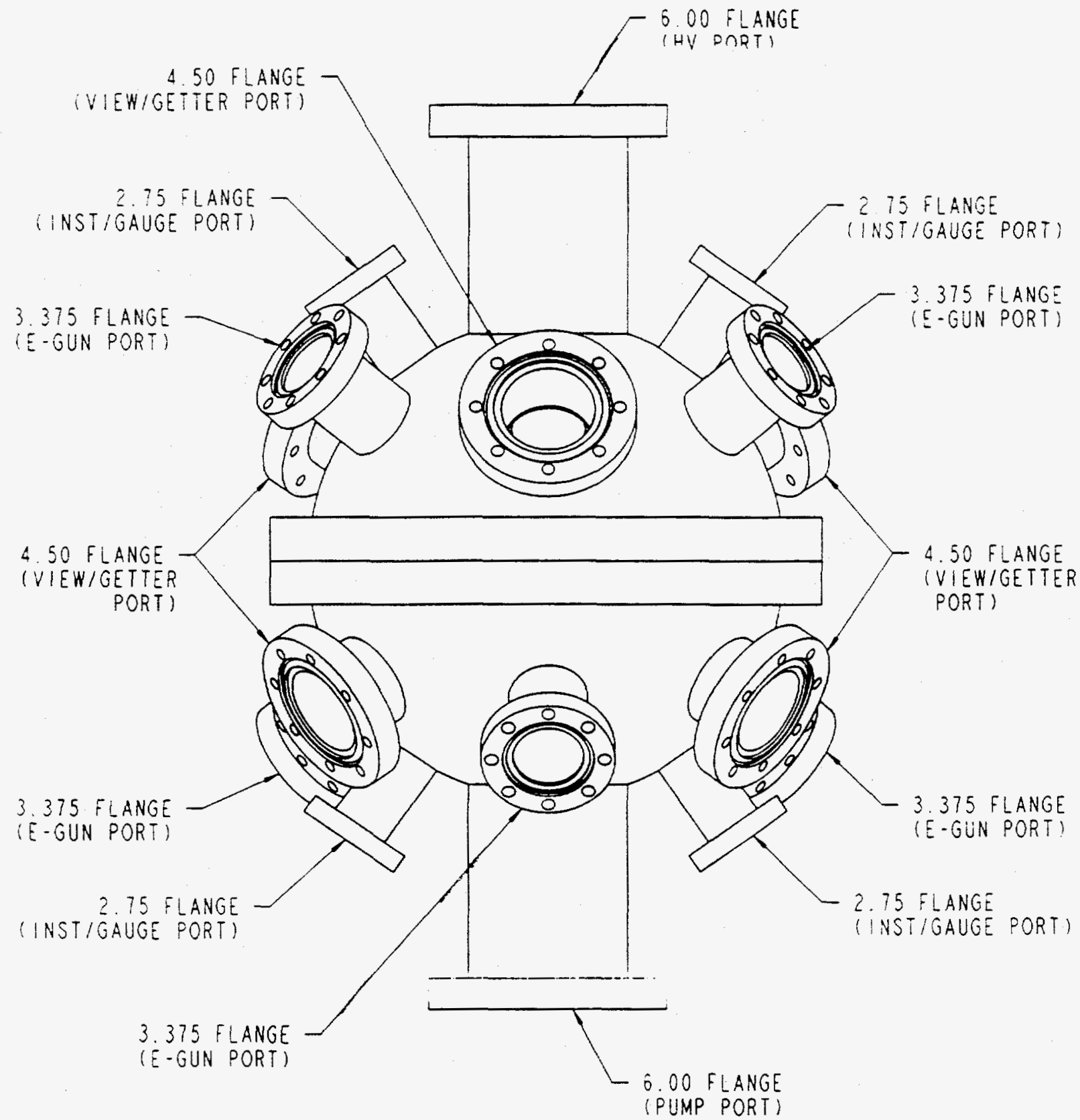

Figure 5. Front view of vacuum chamber of base-line design IEC neutron source. This source is scheduled to be fabricated under a follow-on project to this LDRD study. 